\title{
The crazy-paving pattern: a radiological-pathological correlation
}

\author{
Walter De Wever • Joke Meersschaert • Johan Coolen • \\ Eric Verbeken • Johny A Verschakelen
}

Received: 1 June 2010 /Revised: 8 November 2010 /Accepted: 16 December 2010 /Published online: 9 January 2011

(C) European Society of Radiology 2011

\begin{abstract}
The crazy-paving pattern is a linear pattern superimposed on a background of ground-glass opacity, resembling irregularly shaped paving stones. The crazypaving pattern is initially described as the pathognomonic sign of alveolar proteinosis. Nowadays this pattern is a common finding on high-resolution CT imaging, and can be seen in a number of acute and chronic diseases. The purpose of this paper is to illustrate different diseases that cause this crazy-paving pattern and to correlate the radiological findings from computed tomography with the histopathological findings.
\end{abstract}

Keywords Crazy-paving pattern · Ground-glass opacity · Linear pattern $\cdot$ Interlobular septa,interstitium

\section{Introduction}

The superimposition of a linear pattern on ground-glass opacity on computed tomography images results in a pattern that is termed crazy-paving pattern, resembling the structure of irregularly shaped paving stones $[1,2]$. The crazy-paving pattern is a common finding on thin-section computed tomography (HRCT), but also on multidetector computed tomography (MDCT). Ground-glass opacity is

\footnotetext{
W. De Wever $(\bowtie) \cdot J$. Meersschaert $\cdot$ J. Coolen $\cdot$

J. A. Verschakelen

Department of Radiology, University Hospitals Leuven,

Herestraat 49,

3000 Leuven, Belgium

e-mail: walter.dewever@uzleuven.be

E. Verbeken

Department of Histopathology, University Hospitals Leuven,

Herestraat 49,

3000 Leuven, Belgium
}

defined as a hazy increase in lung density with preservation of airway and vessel margins [3]. Ground-glass opacity occurs when there is a mild decrease in the amount of air in the airspaces and a filling of the airspaces with fluid, cells or other material, thickening of the alveolar walls or thickening of the interstitium. The linear component of this pattern can be caused by a thickening of the interlobular septa (septal lines), a thickening of the intralobular septa and the intralobular interstitium (intralobular reticular pattern and intralobular branching lines) or a linear deposition of material within the airspaces at the borders of the acini (periacinar pattern) (Fig. 1) [4]. The crazypaving pattern was initially described as a pathognomonic sign of alveolar proteinosis; however, nowadays, this pattern has been reported in a variety of acute and chronic diseases as summarised in Table 1 [2, 5-8]. The purpose of this paper is to illustrate different diseases showing a crazypaving pattern. The diagnosis is made based on clinical or on histological findings. If histopathological proof is available, a radiological-histopathological correlation is made.

\section{Materials and methods}

A retrospective review of the medical records of our radiological computed tomography database was performed, from 1 January 2008 until 31 December 2008, searching for patients reported to have a "crazy-paving" pattern on a CT of the chest. In total, 98 patients with a crazy-paving pattern were retained and reviewed. To rule out acute pulmonary embolism, most of the patients underwent interstitial pathological features or underwent their chest $\mathrm{CT}$ in an oncological setting. All these patients underwent a dedicated MDCT of the chest with $100 \mathrm{mAs}$, 


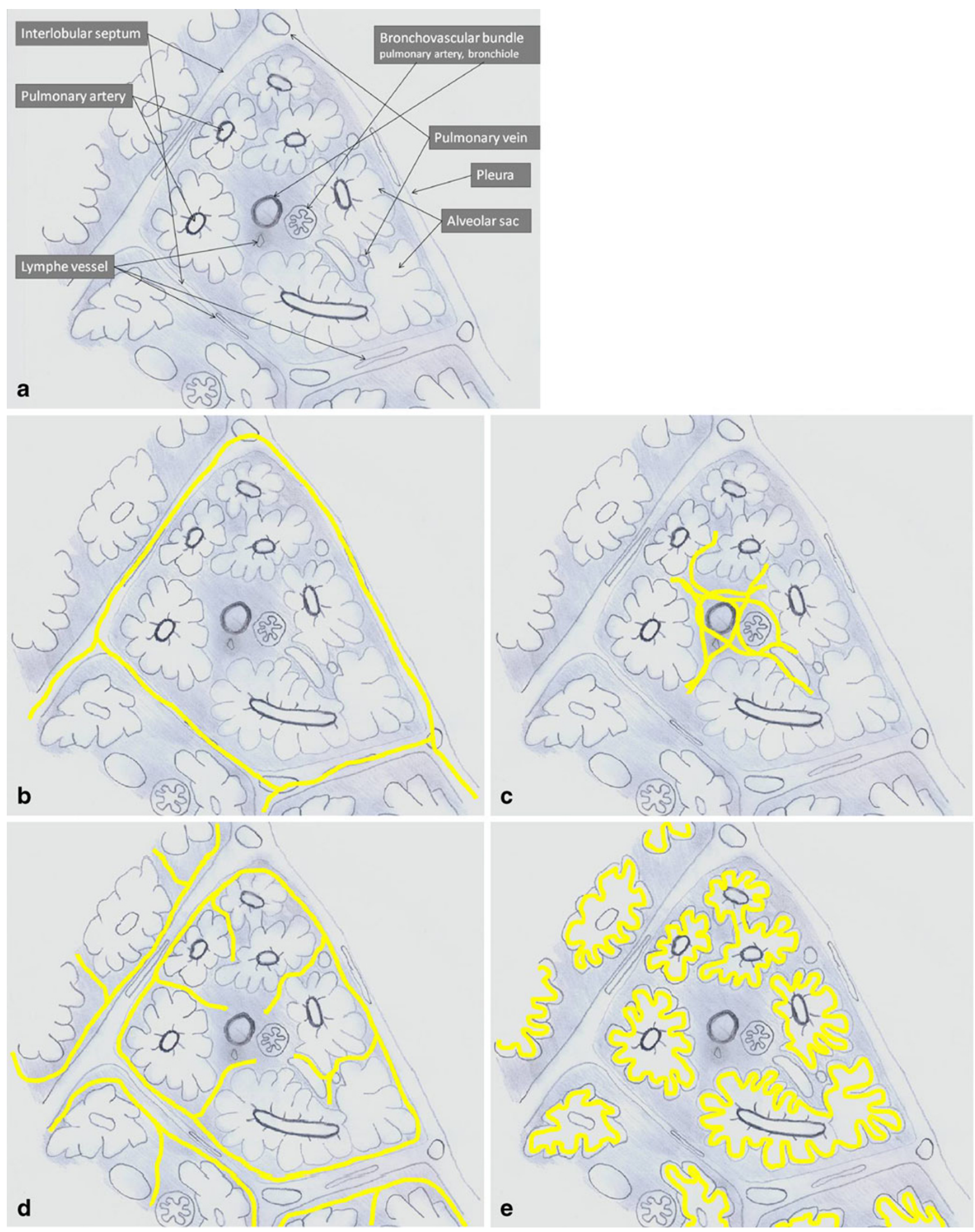

Fig. 1 a Anatomy of the secondary pulmonary lobule. b-e The reticular pattern: $\mathbf{b}$ thickening of the interlobular septa; $\mathbf{c}$ thickening of the intralobular interstitium; d irregular areas of fibrosis; e periacinar pattern 
Table 1 Differential diagnosis of the crazy-paving pattern

\begin{tabular}{ll}
\hline Acute diseases & Subacute/chronic diseases \\
\hline Pulmonary oedema & Usual interstitial pneumonia (UIP) \\
Pulmonary infection (bacterial, viral, pneumocystis jiroveci, mycoplasma) & Non-specific interstitial pneumonia (NSIP) \\
Pulmonary haemorrhage & Alveolar proteinosis \\
Acute interstitial pneumonia (AIP) & Organising pneumonia \\
Adult (acute) respiratory distress syndrome (ARDS) & Vasculitis (Churg-Strauss syndrome) \\
Radiation pneumonitis & Eosinophilic pneumonia (chronic) \\
Eosinophilic pneumonia & Tumour \\
& Lymphangitic spread of tumour \\
& Sarcoidosis \\
& Lipid pneumonia \\
& Alveolar microlithiasis
\end{tabular}

$120 \mathrm{kV}$, slice thickness of 1 and 3 or $5 \mathrm{~mm}$, and table feed of $12 \mathrm{~mm}$ per rotation, with or without intravenous contrast administration, according to the indication of chest CT. Only seven patients with a crazy-paving pattern on chest CT also underwent an open lung biopsy to make the definitive diagnosis. In 59 patients, the definitive diagnosis was made on a clinical basis. In the remaining 32 patients, the cause of the crazy-paving pattern remained undecided, because patients were not followed further in our institution.

\section{Results}

Ninety-eight patients with a crazy-paving pattern were retained and reviewed. Table 2 summarises the different causes of the crazy-paving pattern as found on open lung biopsy or based on clinical decision. Only seven patients underwent open lung biopsy to establish the diagnosis.

\section{Patient 1}

A 46-year-old man presented with a 1-week history of progressive dyspnoea. He also complained of a cough and the production of white mucus in the morning. He reported a smoking habit of one pack of cigarettes per day with no further information regarding his past smoking history.

Chest radiograph and CT were undertaken. Chest radiograph (Fig. 2a) showed a reticular pattern more pronounced in the central parts of the lungs. There was also an increase in lung density centrally in both lungs. No pleural fluid was noted, and the heart and central vascular structures were normal. On CT, there was a patchy distribution of areas with increased lung attenuation throughout both lungs. Superimposed on this increased lung attenuation a linear pattern was seen. There were multiple small regular and irregular lines. Some of them were thickened interlobular septa. More lines were visible in the centre of the secondary pulmonary lobule in a very irregular pattern suggesting thickening of the intralobular interstitium.

Histopathological evaluation of a specimen from open lung biopsy out of the right lung showed amorphous eosinophilic material in the alveoli, positive on periodic acid Schiff (PAS) staining. This eosinophilic material

Table 2 Different causes of crazy-paving pattern in our database

\begin{tabular}{ll}
\hline Aetiology & Number (n) \\
\hline Infection & \\
-Bacterial infection & 10 \\
-Viral infection & 3 \\
-Fungal infection & 4 \\
-Non-specified infection & 10 \\
ARDS & 6 \\
Acute pulmonary oedema & 5 \\
Interstitial lung disease (UIP, NSIP,AIP, EAA, MTCD) & 16 \\
Tumour & 2 \\
Lymphangitis carcinomatosa & 1 \\
Radiation pneumonitis & 3 \\
Sarcoidosis & 2 \\
Alveolar proteinosis & 1 \\
Graft-versus-host disease & 1 \\
Lipid pneumonia & 1 \\
Organising pneumonia & 1 \\
Undecided & 32 \\
\hline ARDS: Acut &
\end{tabular}

ARDS: Acute respiratory distress syndrome

UIP: Usual interstitial pneumonia

NSIP: Non-specific interstitial pneumonia

AIP: Acute interstitial pneumonia

EAA: Extrinsic allergic alveolitis

MTCD: Mixed tissue connective disease 
Fig. 2 Alveolar proteinosis. a Chest radiograph showed a reticular pattern that was most pronounced in the central parts of the lungs. There was also a decrease in the lung translucency centrally in both lungs. Heart and central vessels were normal.

There was no pleural effusion. b On CT, a patchy distribution of a crazy-paving pattern was visible. The lines corresponded to a deposition of material within the airspaces at the borders of the acini (1) in the secondary pulmonary lobules, but also along the interlobular (2) and intralobular septa (3): the periacinar pattern. c

Radiological-histopathological correlation. Histopathological evaluation of a specimen out of the right lung showed amorphous eosinophilic material in the alveoli (*) positive on periodic acid Schiff (PAS) staining. This material corresponded to deficient surfactant. Filling of the alveoli $(*)$ was responsible for the ground-glass appearance on CT. When the airspaces adjacent to the inter- and intralobular septa (black arrow) and to the alveolar walls filled, the periacinar pattern became visible
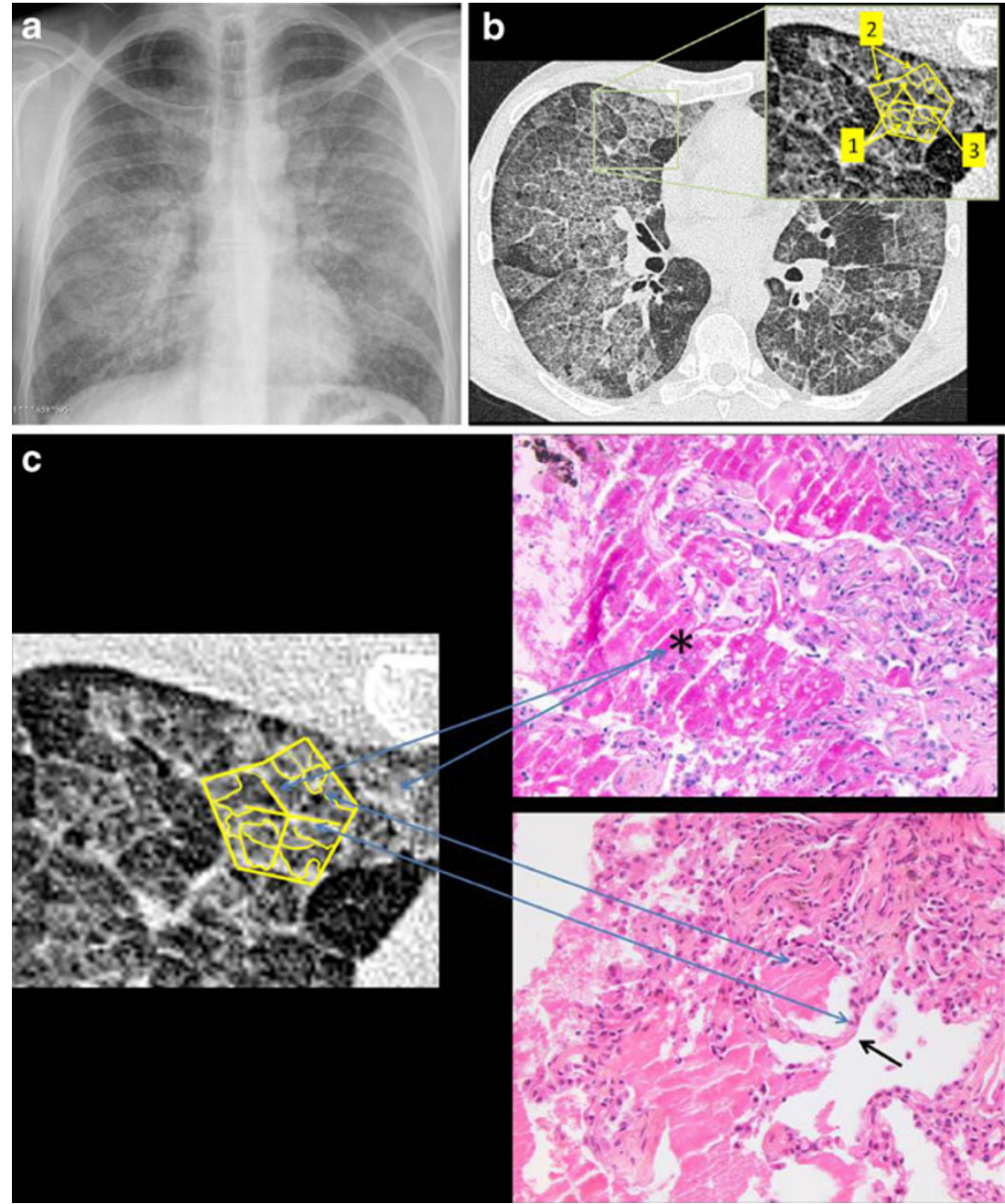

corresponded with deficient surfactant (Fig. 2c). The lines visible on CT corresponded to deposition of material within the airspaces at the borders of the acini in the secondary pulmonary lobules (periacinar pattern; Fig. 2b).

The diagnosis of alveolar proteinosis was made.

\section{Patient 2}

A 62-year-old woman with progressive shortness of breath on exercise.

Chest radiograph and CT were undertaken. Chest radiograph showed a patchy distribution of areas with increased lung density (Fig. 3a). There was also an increase in linear markings in both lungs. On CT, a crazy-paving pattern was seen with a geographic distribution. Some of the lines were thickened interlobular septa. Centrally in the secondary pulmonary lobule we could also see a spider of lines: thickening of the intralobular septa. These findings were seen predominantly in the upper lung areas (Fig. $3 \mathbf{b}$ ).

Although the patient had no history of bird exposure, serum precipitins against pigeons were elevated. To resolve this paradox, an open long biopsy was performed. Histology demonstrated interstitial pneumonia with lymphocytes, plasma cells and foamy macrophages in the interstitium. Epithelioid granulomas without caseation were also seen. There was no fibrosis (Fig. 3c).

The diagnosis of hypersensitivity pneumonitis was made.

\section{Patient 3}

An 80-year-old man with rapidly progressive dyspnoea.

A chest radiograph and $\mathrm{CT}$ were undertaken. The chest radiograph showed a patchy distribution of areas with consolidation. There was also a fine reticular pattern, most pronounced in the periphery of both lungs (Fig. 4a). Chest CT, performed to rule out acute pulmonary embolism, was negative for the presence of lung emboli. A crazy-paving pattern with a scattered distribution of ground-glass opacities and a linear pattern superimposed, with multiple small irregular lines, was visible. Traction bronchiectasis was seen in the periphery of both lungs (Fig. 4b). 

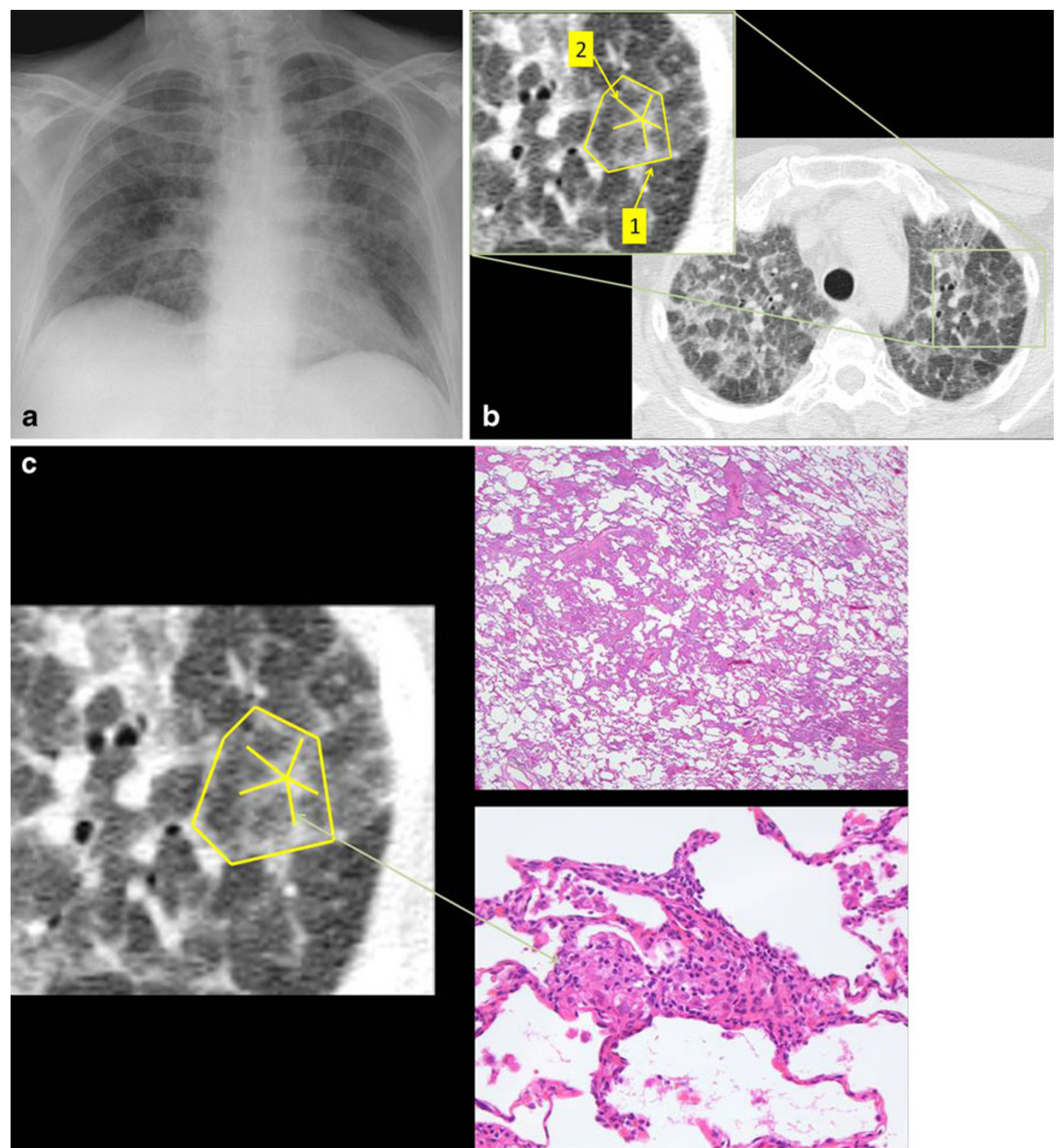

Fig. 3 Hypersensitivity pneumonitis. a Chest radiograph showed patchy distribution of areas with increased lung density. There was also an increase in the linear pattern in both lungs. b On CT, a crazy-paving pattern was seen with a geographic distribution of ground-glass opacities with the superimposition of thickened inter- (1) and intralobular (2) septa. The findings were seen predominantly in the upper lung areas. c
Radiological-histopathological correlation. Histology demonstrated interstitial pneumonia with lymphocytes, plasma cells and foamy macrophages in the interstitium. Epithelioid granulomas without caseation were also seen. There was no fibrosis. The alterations in the walls of the alveoli and the inflammation in the interstitium were visible as thickening of the inter- and intralobular lines
On histology, thickening of the interstitium with variable degrees of severity was seen, leaving some alveolar septa almost completely normal, whereas others were thickened. Fibrinous exudates, honeycombing and mild inflammatory alveolitis were also present (Fig. 4c).

The diagnosis of usual interstitial pneumonia (UIP) was made.

\section{Patient 4}

A 56-year-old woman with increasing dyspnoea.

A chest radiograph and CT were undertaken. The chest radiograph showed a reticulation of the lung parenchyma, diffusely spread in both lungs, centrally and peripherally (Fig. 5a). Chest CT showed a crazy-paving pattern especially in the periphery of both lungs. There was an increase in lung 
Fig. 4 Usual interstitial

pneumonia. a Chest radiograph showed patchy distribution of areas with consolidation and a fine reticular pattern, most pronounced in the periphery of both lungs. b A crazy-paving pattern was visible with scattered distribution.

Superimposed on the ground-glass opacities a linear pattern with multiple small irregular lines was visible (intralobular fibrosis) (1).

Traction bronchiectasis was seen in the periphery of both lungs (white arrow). c Radiologicalhistopathological correlation. On histology, thickening of the interstitium (arrow) with variable severity was seen, leaving some alveolar septa almost completely normal, whereas others were thickened. Fibrinous exudates, honeycombing $(*)$ and mild inflammatory alveolitis were also present
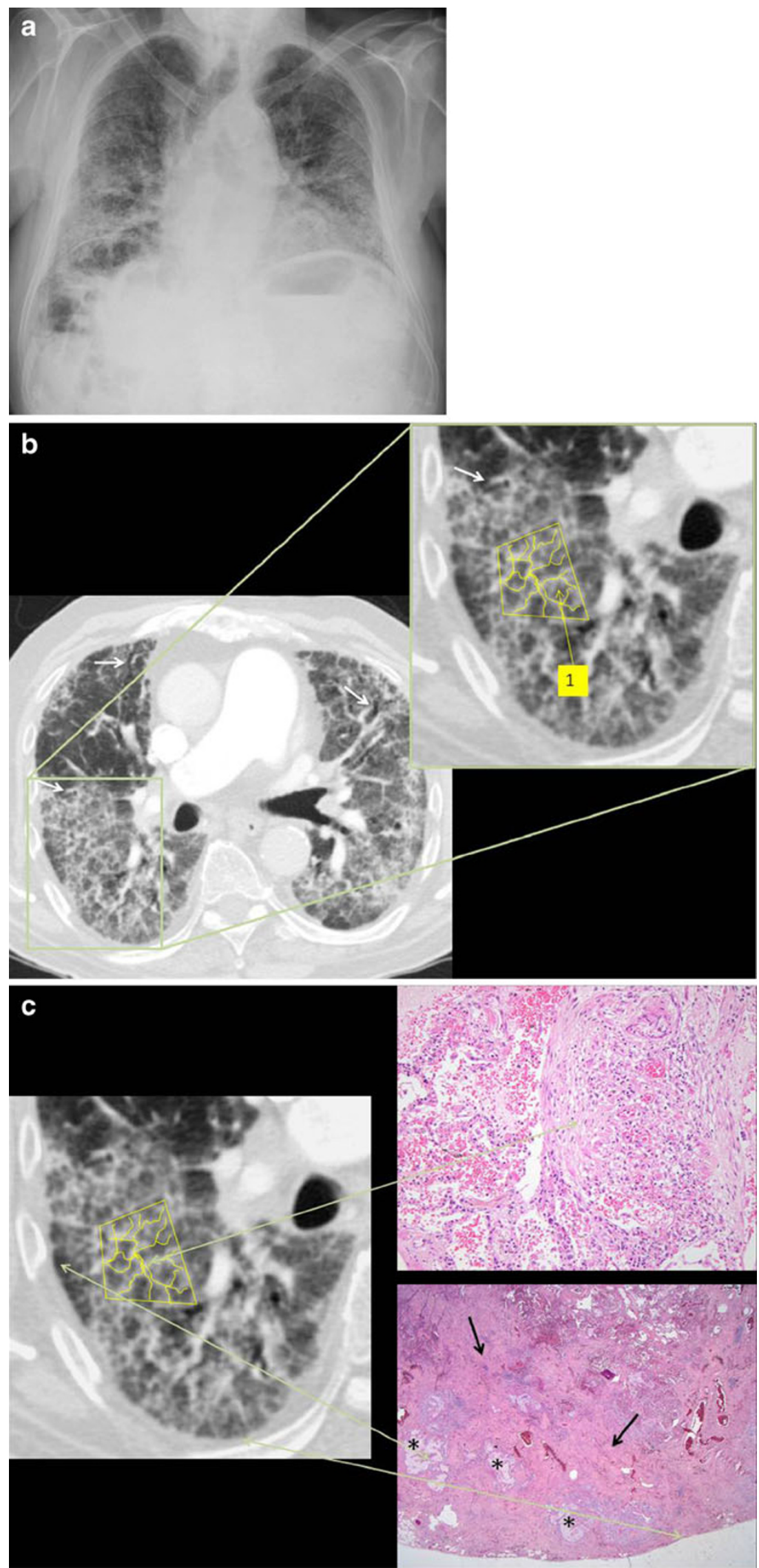
Fig. 5 Non-specific interstitial pneumonia. a Chest radiograph showed reticulation in the lung parenchyma, diffusely spread in both lungs, centrally and peripherally. b Chest CT showed a crazy-paving pattern especially at the periphery of both lungs. There was an increase in lung attenuation (ground-glass opacification) with a

superimposition of a reticular pattern with thickening of the inter- (1) and intralobular (2) septa. c Radiologicalhistopathological correlation. Histological evaluation showed a homogeneous fibrotic thickening of the interstitium with inflammation.

Macrophages were visible within the alveolar septa Homogeneous interstitial inflammation was seen, corresponding to the diffuse ground-glass opacities, whereas fibrosis in the interstitium and alveolar septa (black arrow) was related to the superimposed linear pattern
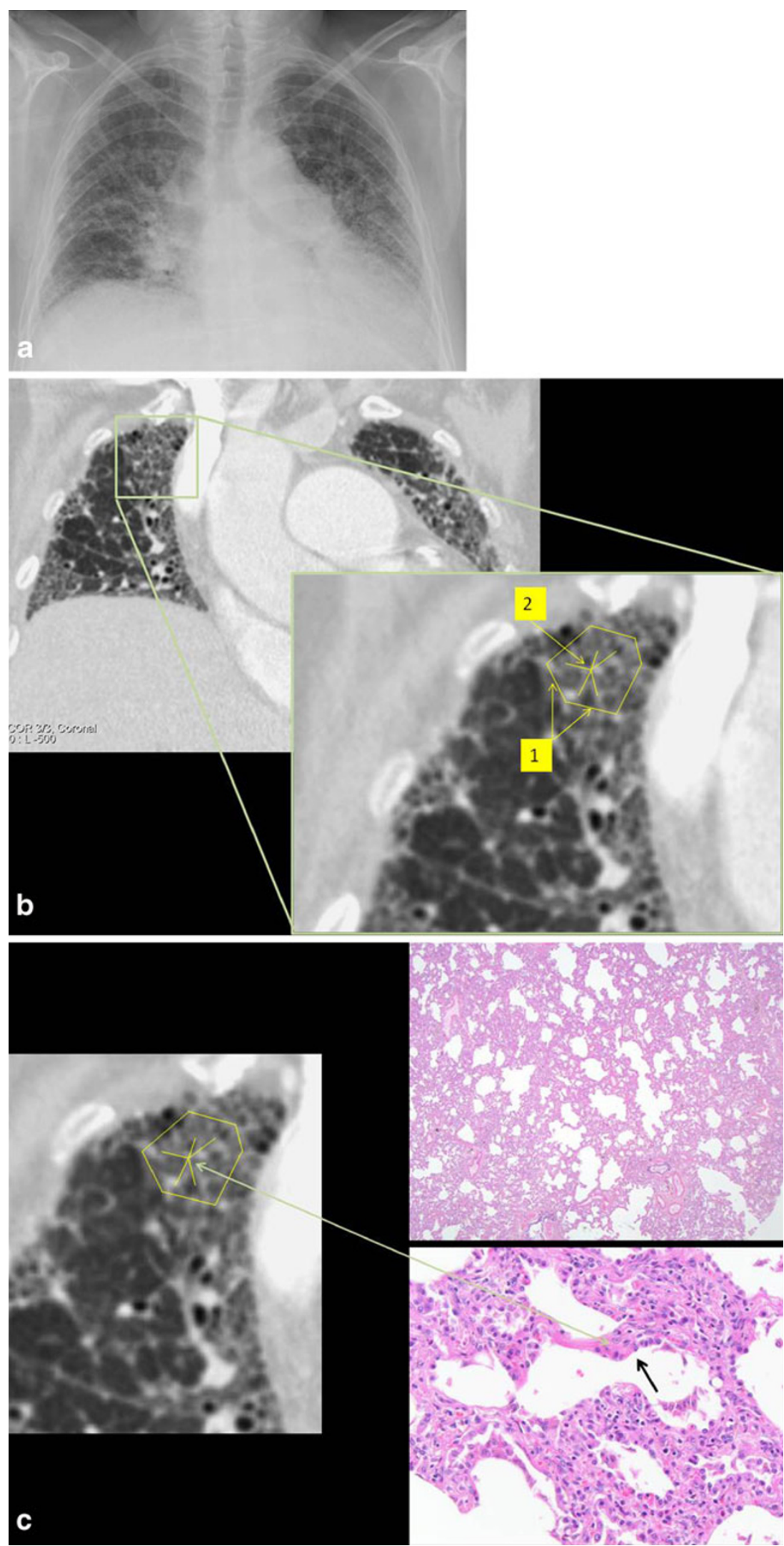
Fig. 6 Radiation pneumonitis. a Chest radiograph showed an area of consolidation in the right lung with an air bronchogram. There was also loss of volume of the right lung. b CT showed the therapy response of the tumour. There was patchy distribution of a crazy-paving pattern with increased lung attenuation (ground-glass opacity) and thickening of the interlobular septa in the right lung (1). c Radiological-

histopathological correlation. Histological examination after autopsy showed airspace filling with an exudate in combination with thickening of the interlobular septa (arrow), thickening of the interstitium surrounding the airspaces and also the presence of irregular fibrosis (dotted arrow). Alveolar spaces filled with an exudate of proteinaceous material were responsible for the ground-glass opacities on CT. The reticular pattern was due to congestion of capillaries and oedema of the interstitium
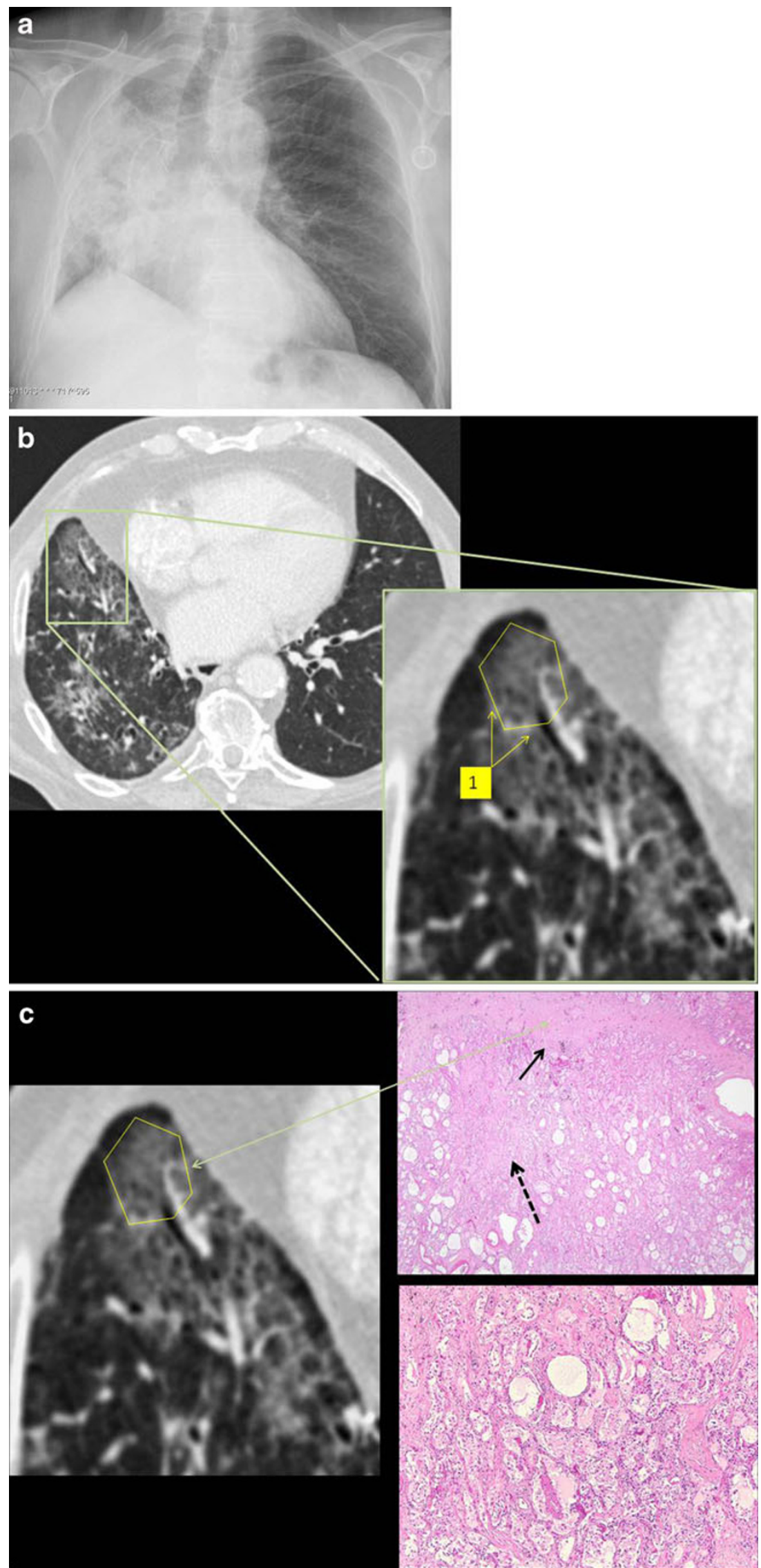
Fig. 7 Exogenous lipid pneumonia. a Chest radiograph showed a decrease in lung translucency in the caudal region of the right lung with an air bronchogram. b Chest CT showed a crazy-paving pattern with areas of increased lung attenuation and with thickening of interlobular septa (1), even thickening of the intralobular interstitium (2). c Radiologicalhistopathological correlation. Histological examination showed alveoli filled with lipid particles $(*)$, some ingested in macrophages $(+)$ with the formation of lipid granulomas
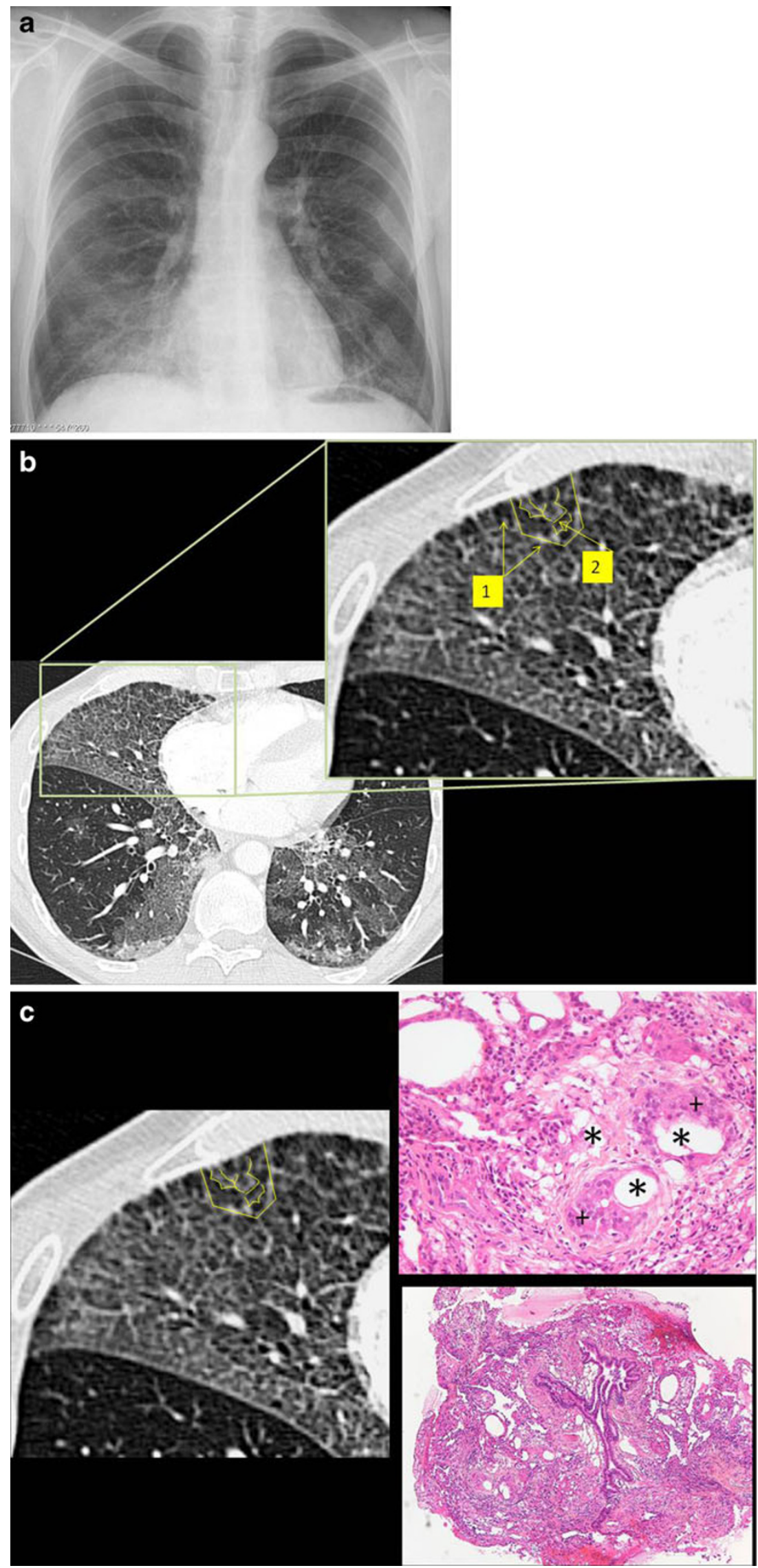
Fig. 8 Lymphangitic carcinomatosis. a Chest radiograph showed a pleural effusion in the right haemothorax. An increased linear pattern was seen in the left and right upper lung. b CT showed a diffuse crazy-paving pattern with areas of groundglass attenuation and thickening of the interlobular septa (1). There were also some small nodular lesions visible mostly in the left upper lobe suggestive of pulmonary metastases (2). c Radiological-histopathological correlation. Histological examination of the autopsy specimen demonstrated thickening of the interlobular septa (*) due to fibrosis and the presence of tumour cells. There was also perivascular (arrow) thickening due to an expansion of lymphatic spaces by tumour cells. The histological reaction was that of diffuse alveolar damage and consisted of hyaline membranes in the alveolar ducts and respiratory bronchioles while the alveolar spaces fill with an exudate of proteinaceous material. This corresponded to the ground-glass opacities on CT. The reticular pattern was due to congestion of capillaries and oedema of the interstitium
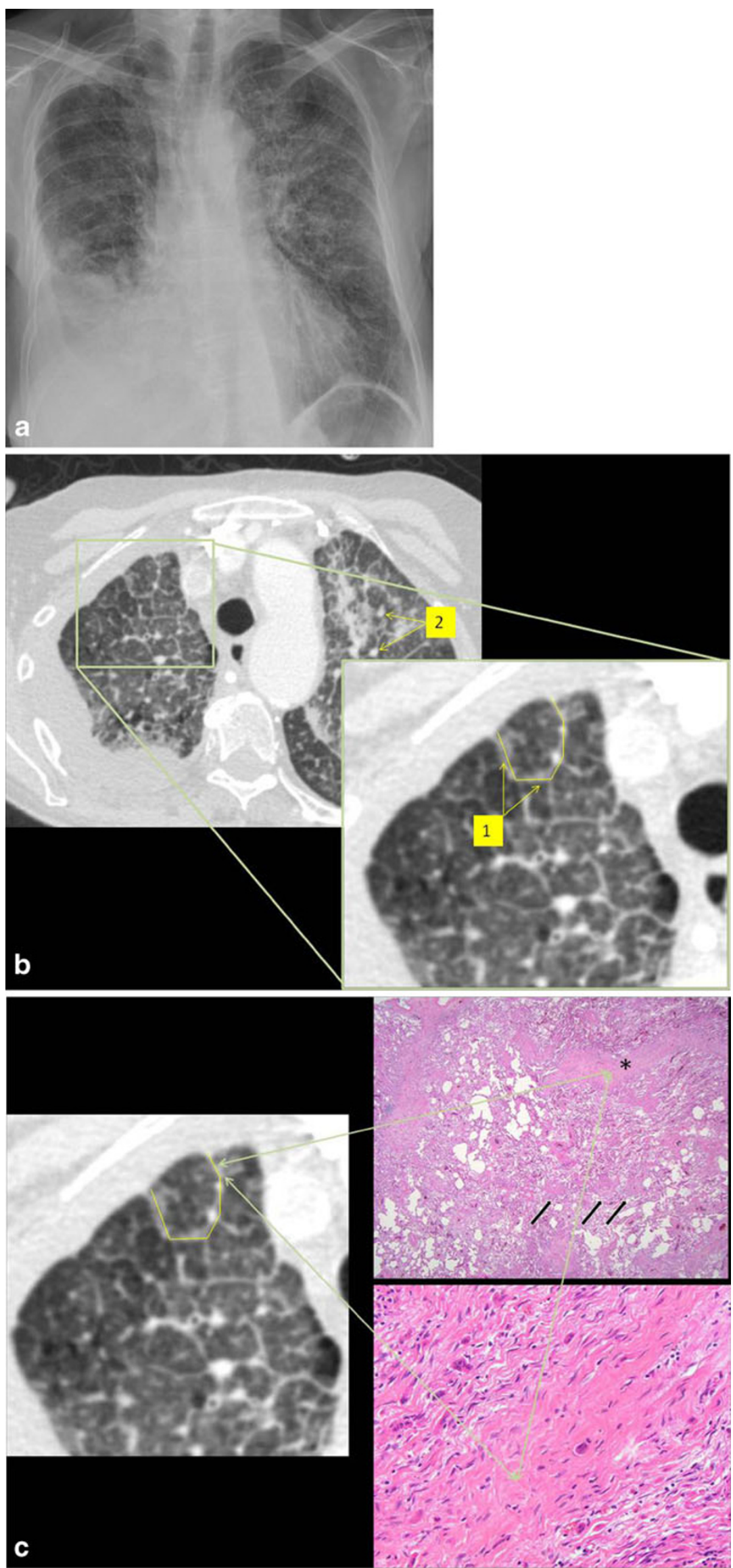

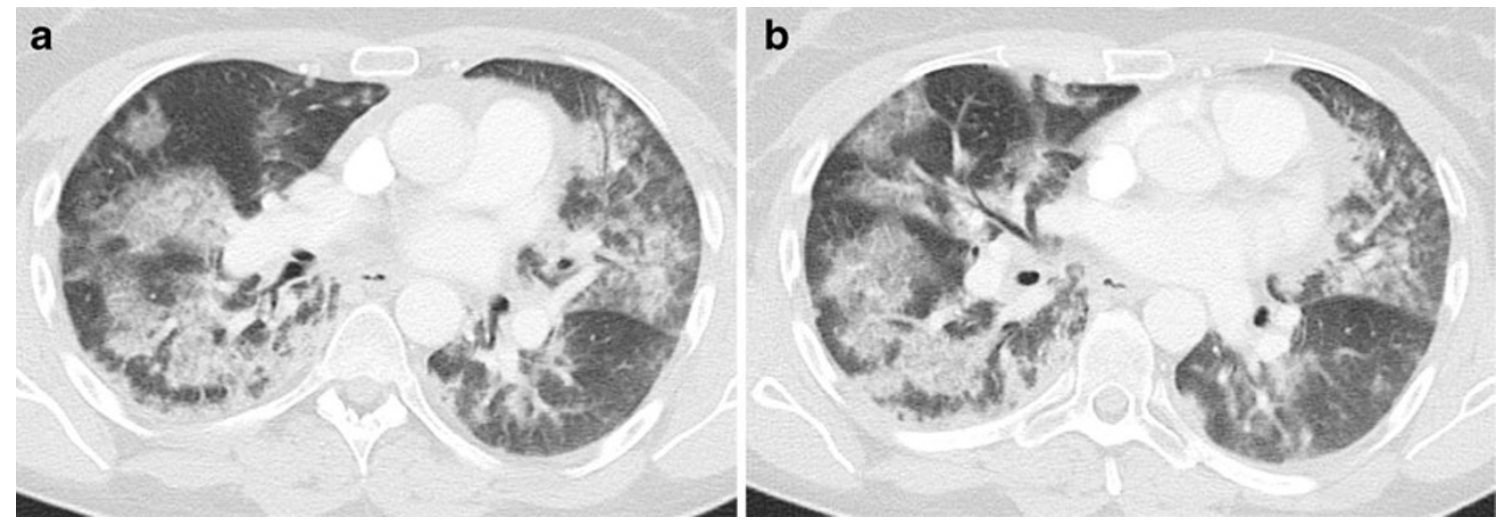

Fig. 9 Pneumocystis jirovecii infection. CT showed a patchy distribution of areas with ground-glass opacification in both lungs, more pronounced in central parts, with a superimposition of a linear pattern

attenuation (ground-glass opacification) with a superimposition of thickened inter- and intralobular septa (Fig. 5b).

Histological evaluation showed a homogeneous fibrotic thickening of the interstitium with inflammation. Macrophages were visible within the alveolar septa (Fig. 5c).

The diagnosis of non-specific interstitial pneumonia (NSIP) was made.

\section{Patient 5}

A 71-year-old man with a limited small cell lung cancer developed fever and a cough after radiation therapy.

A chest radiograph and CT were undertaken. The chest radiograph showed an area of consolidation in the right lung with an air bronchogram. There was also loss of volume of the right lung (Fig. 6a). CT showed a decrease in the size of the tumour consistent with response to therapy. There was a patchy distribution of a crazy-paving pattern with ground-glass opacities and thickening of the interlobular and intralobular septa (Fig. 6b).

Histological examination after autopsy showed airspace filling with an exudate in combination with thickening of the interlobular septa, thickening of the interstitium sur- rounding the airspaces and also the presence of irregular fibrosis (Fig. 6c).

The diagnosis of radiation pneumonitis was made.

\section{Patient 6}

A 54-year-old man with progressive dyspnoea.

A chest radiograph and CT were undertaken. The chest radiograph showed decreased translucency with an air bronchogram in the right lower lobe. There were no signs of interstitial lung disease (Fig 7a). Chest CT showed a crazypaving pattern with areas of increased lung attenuation and with thickening of the interlobular septa, even thickening of the intralobular interstitium in the right middle and lower lobe (Fig. 7b). Histological examination showed alveoli filled with lipid particles, some of them ingested in macrophages with the formation of lipid granulomas (Fig.7c).

The diagnosis of exogenous lipid pneumonia was made.

\section{Patient 7}

A 73-year-old woman with an insidious onset of unexplained and progressive dyspnoea.
Fig. 10 Acute respiratory distress syndrome. CT revealed bilateral areas with ground-glass attenuation superimposed with a reticular pattern. These lines corresponded to thickening of the interlobular septa, but also thickening of the intralobular interstitium
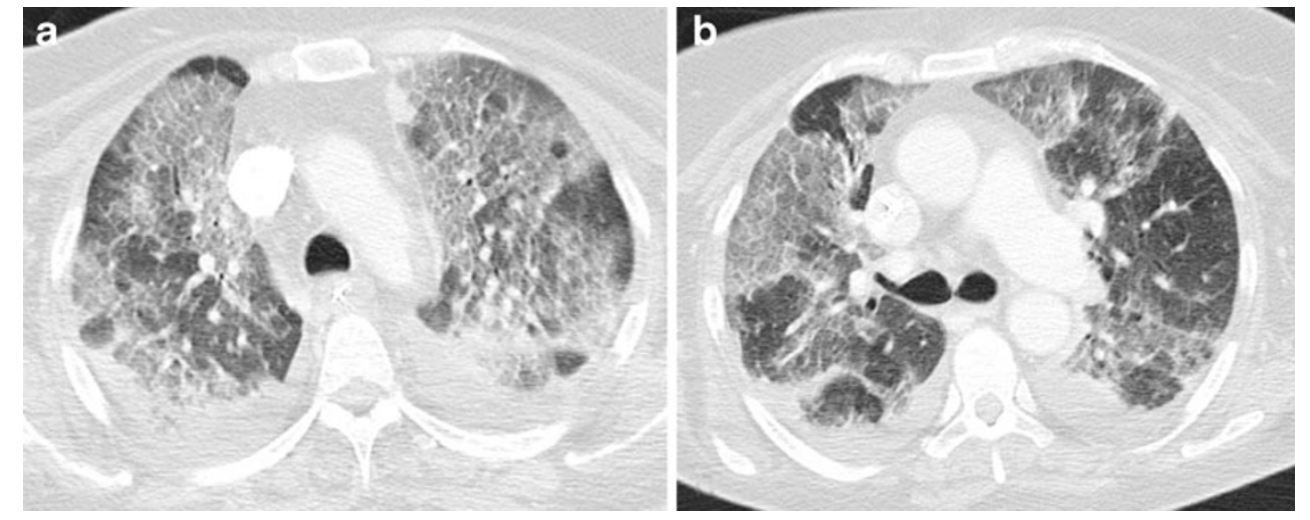
Fig. 11 Pulmonary oedema. CT showed patchy distribution of areas with ground-glass opacification and a linear pattern. Most of the lines were thickened interlobular septa. Within the secondary pulmonary lobule, enlarged vascular structures with a spider configuration were seen. There were also some other intralobular lines
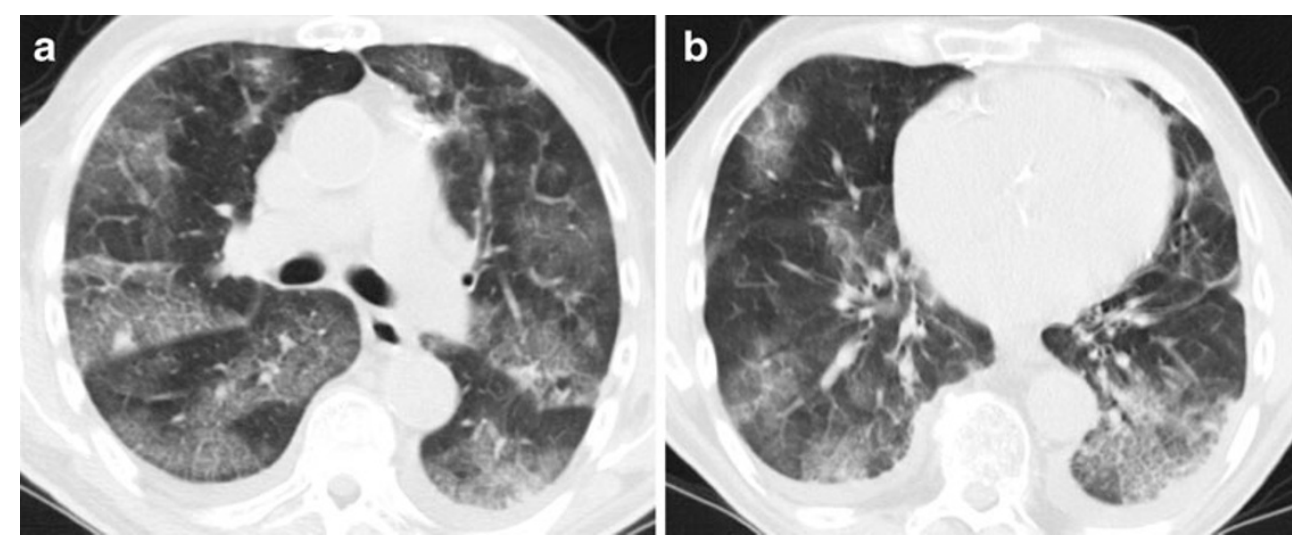

A chest radiograph and $\mathrm{CT}$ were undertaken. The chest radiograph showed a pleural effusion in the right hemothorax. An increased reticular pattern was seen in the left upper lung field and to a lesser degree also in the right upper lung field (Fig. 8a). CT showed a diffuse crazypaving pattern with areas of ground-glass attenuation and thickening of the interlobular septa. There were also some small nodular lesions visible, mostly in the left upper lobe, suggestive of pulmonary metastases (Fig. $8 \mathbf{b}$ ).

Histological examination of the autopsy specimen demonstrated heterogeneous thickening of the interlobular septa due to fibrosis and the presence of tumour cells. There was also perivascular thickening due to an expansion of lymphatic spaces by tumour cells.

The diagnosis of lymphangitic carcinomatosis was made.

\section{Patient 8}

A 34-year-old woman with thrombotic thrombocytopaenic purpura and severe myasthaenia gravis developed progressive respiratory insufficiency. A chest CT was undertaken. CT showed a patchy distribution of areas with ground-glass opacification in both lungs, more pronounced in the central parts of both lungs (Fig. 9). There was also a superimpo- sition of a linear pattern. Most of the lines were thickened interlobular septa.

The diagnosis of pneumocystis jirovecii pneumonia was made based on clinical and laboratory findings.

\section{Patient 9}

A 67-year-old woman who received a total knee prosthesis developed septic shock with ARDS in the postoperative period. A chest CT was undertaken (Fig. 10). This CT revealed bilateral areas with ground-glass attenuation superimposed with thickened interlobular septa but also thickening of the intralobular interstitium.

\section{Patient 10}

An 83-year-old man with the diagnosis of acute lymphatic leukaemia developed cardiac decompensation with oedema of the lower limbs. CT of the chest (Fig. 11) showed a patchy distribution of areas with ground-glass opacification. A superimposed linear pattern was also present. Most of the lines were thickened interlobular septa. Within the secondary pulmonary lobule, enlarged vascular structures with a spider configuration were seen. There were also some other intralobular lines.

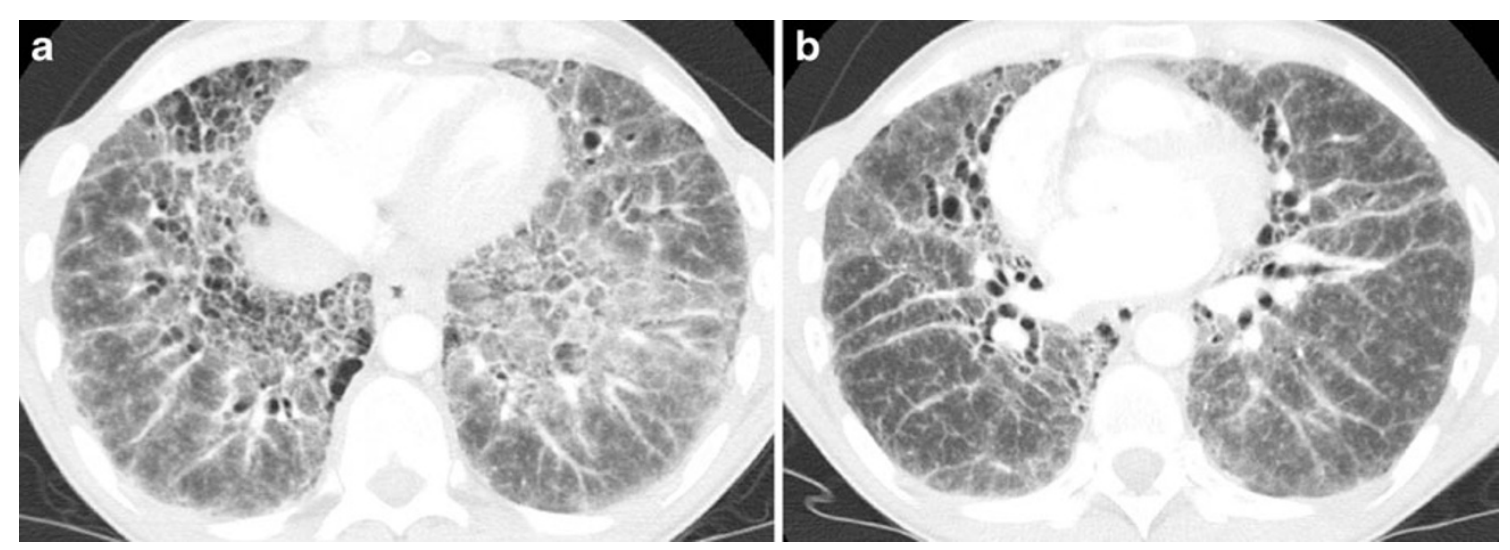

Fig. 12 Sarcoidosis. CT showed a diffuse increase in lung attenuation (ground-glass attenuation) with the superimposition of an irregular reticular pattern: thickening of the interstitium and thickening of the peribronchovascular interstitium 
Fig. 13 Graft-versus-host disease. CT revealed multiple areas of ground-glass attenuation and consolidations. There was also a superimposition of multiple lines: thickened inter- and intralobular septa and intralobular fibrosis
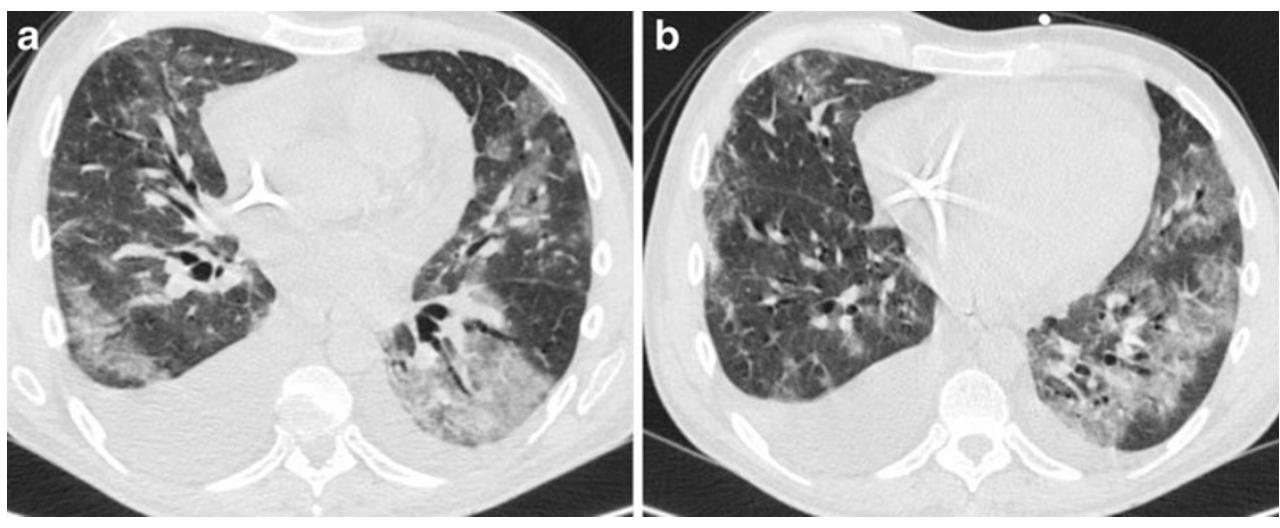

\section{Patient 11}

A 44-year-old man with sarcoidosis underwent a control CT of the chest. There was diffuse increased lung attenuation with the superimposition of multiple irregular lines and also irregular thickening of the bronchovascular bundles: the crazy-paving pattern (Fig. 12). Interstitial fibrosis was the cause of the irregular thickening of the interstitium.

\section{Patient 12}

A 40-year-old man with haematopoietic stem cell transplantation. He developed dyspnoea, and CT was undertaken. CT revealed multiple areas of ground-glass attenuation and consolidations. There was also a superimposition of multiple lines: thickened inter- and intralobular septa in intralobular lines caused by fibrosis (Fig. 13).

The diagnosis of graft-versus-host disease was made.

\section{Patient 13}

A 24-year-old woman with bilateral lung transplantation. A control CT was performed and showed patchy distribution of areas of ground-glass opacification with the superimpo- sition of thickened interlobular septa: the crazy-paving pattern (Fig. 14).

The diagnosis of organising pneumonia was made on a clinical basis.

\section{Patient 14}

A 75-year-old man known to have bronchioloalveolar carcinoma. Chest CT showed a patchy distribution of areas with increased density, areas of ground-glass opacification and areas with consolidation. Superimposed on these areas there was a reticular pattern (Fig. 15). These lines correspond to a thickening of the interstitium. The diagnosis was made based on biopsy, which was not performed in our institution.

\section{Discussion}

The crazy-paving pattern is a non-specific pattern. Initially, this pattern was considered to be highly suggestive of alveolar proteinosis. Nowadays, we can find this pattern in different lung diseases: airspace diseases and interstitial diseases [8]. The crazy-paving pattern consists of scattered or diffuse ground-glass attenuation with superimposition of
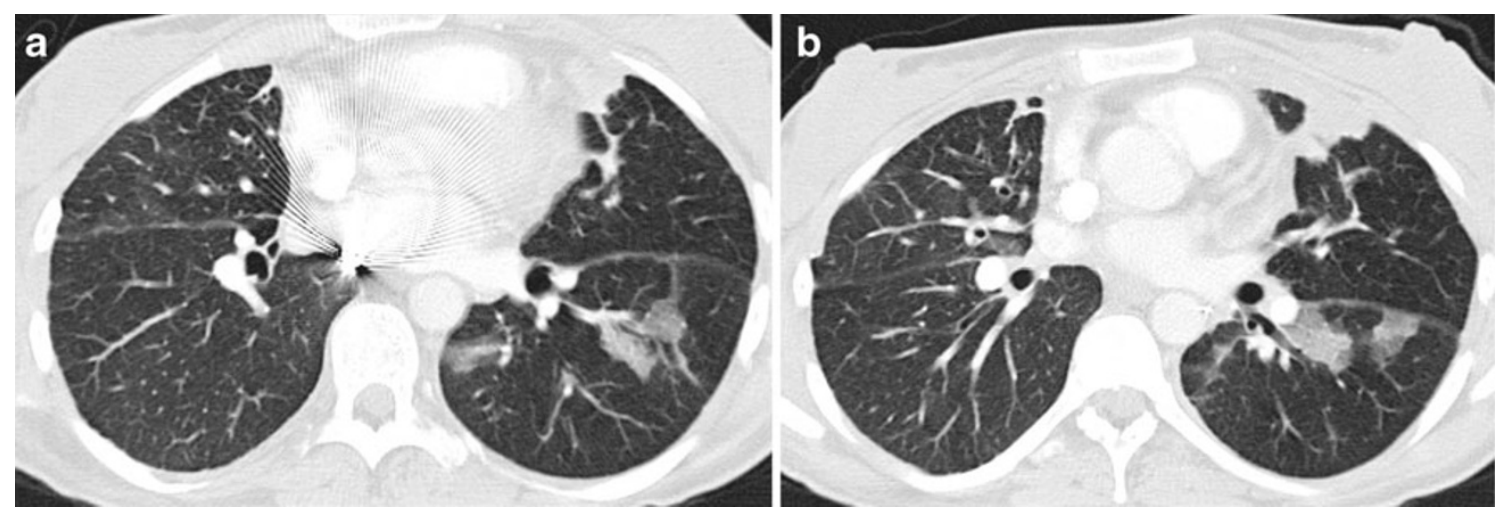

Fig. 14 Organising pneumonia. CT showed patchy distribution of areas of ground-glass opacification with the superimposition of thickened interlobular septa 
Fig. 15 Bronchioloalveolar carcinoma. CT showed patchy distribution of areas with ground-glass opacification and areas with consolidation. Superimposed on these areas there is a reticular pattern corresponding to the thickening of the interstitium

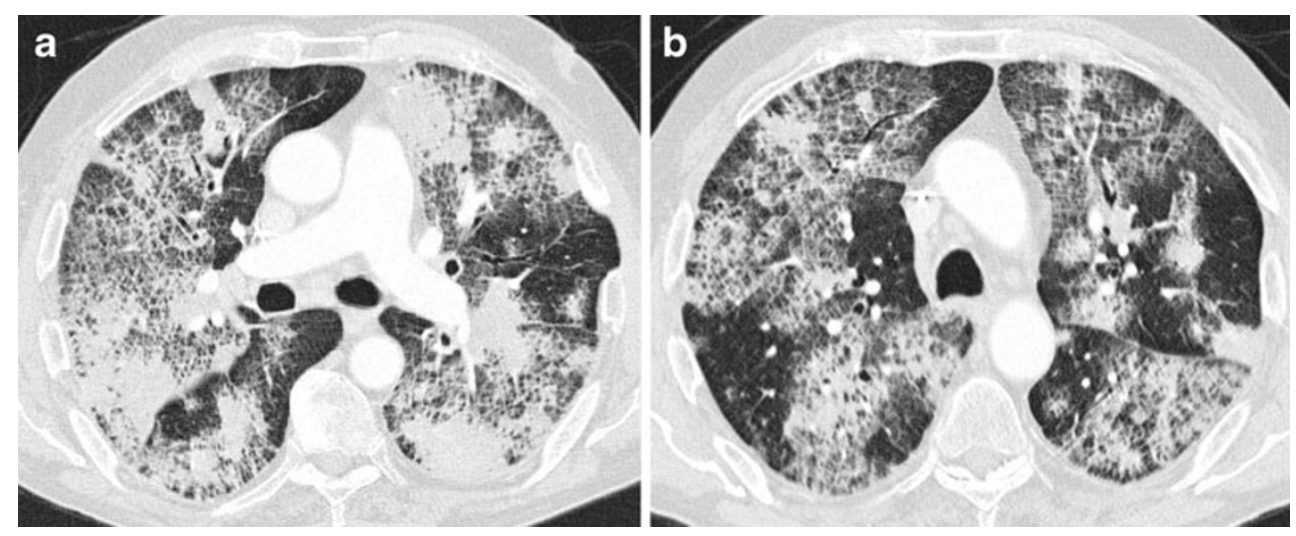

a linear pattern. These lines can be: thickened interlobular septa (septal lines), thickened intralobular septa and thickening of the intralobular interstitium (intralobular reticular pattern and intralobular branching lines), or it can be a linear deposition of material within the airspaces at the borders of the acini and the secondary pulmonary lobules (periacinar pattern) [4].

Alveolar proteinosis and exogenous lipid pneumonia are airspace diseases. In alveolar proteinosis, airspaces are filled with a phospholipoproteinaceous material. On CT, the filling of the alveoli is responsible for the ground-glass appearance. When the airspaces adjacent to the inter- and intralobular septa and to the alveolar walls fill, the periacinar pattern becomes visible (Fig. 2c) [9-11].

Exogenous lipid pneumonia is the result of chronic inhalation of oily substances and is primarily a disease that affects the alveolar spaces. On CT, diffuse ground-glass opacities and consolidations, sometimes with fat attenuation caused by large lipid particles and numerous lipid-laden macrophages distending the alveolar spaces, can be seen, especially in the lower lung areas (Fig. 7c) [9, 12, 13].

Pneumocystis jirovecii pneumonia is a common pulmonary infection in severely immunocompromised patients. Our patient was receiving treatment with Neoral, Imuran, Medrol and Mestinon. Chest radiographs can be normal in up to $18 \%$ of patients. Typical radiographic manifestations on CT are bilateral, perihilar reticular and poorly defined ground-glass opacities with superimposition of lines, which can be associated with interlobular septal thickening [14]. As described by Rossi et al. histological features contributing to the ground-glass attenuation include the foamy nature of the alveolar exudates and thickening of the alveolar walls by oedema and cellular infiltrates [2].

Hypersensitivity pneumonitis, UIP , NSIP, radiation pneumonitis and lymphangitic spread of carcinoma are interstitial diseases. In hypersensitivity pneumonitis, antigen-antibody complexes around the microvasculature cause a neutrophil-rich inflammatory response and subsequent tissue injury. Biopsy in the subacute phase shows heavy infiltrates of lymphocytes and plasma cells in the walls of the alveoli in combination with poorly formed granulomas containing foreign body giant cells. In chronic phases, the interstitial inflammation remains, but fibrosis becomes more apparent and honeycombing can occur. On CT, the alterations in the walls of the alveoli and the inflammation in the interstitium are visible as thickening of the inter- and intralobular lines and thickening of the intralobular interstitium (Fig. 3c) [15].

The cardinal features of UIP on CT include subpleural reticular opacities (intralobular and interlobular septal lines) and honeycombing, increasing from the apex to the base. Ground-glass opacities are inconspicuous or absent in UIP, but focal areas of GGO may be present [16]. On histology, the hallmark is a geographically and temporally heterogeneous parenchymal fibrosis against a background of continuing mild inflammation (Fig. 4c) [17].

In NSIP the predominant finding on HRCT is subpleural, patchy, ground-glass opacification [18]. Traction bronchiectasis, subpleural microcystic honeycombing and irregular linear opacities can be seen in more advanced cases. On histology, homogeneous interstitial inflammation is seen, corresponding to the diffuse ground-glass opacities, whereas fibrosis in the interstitium is related to the superimposed linear pattern (Fig. 5c) [19].

The inflammation of lung tissue, secondary to radiation therapy, is localised in the tissue within the radiation field and depends on the interval since completion of treatment.

In the acute phase (4 to 12 weeks after completion of radiation therapy), the histological reaction is that of diffuse alveolar damage and consists of hyaline membranes in the alveolar ducts and respiratory bronchioles while the alveolar spaces fill with an exudate of proteinaceous material. This corresponds to the ground-glass opacities typically manifesting on CT. The reticular pattern that can be seen in this phase is due to congestion of capillaries and oedema of the interstitium (Fig. 6c) [20].

Pulmonary lymphangitic carcinomatosis is a metastatic lung disease characterised by diffuse spread of tumour to the pulmonary lymphatic system. When tumoral cells spread to the pulmonary lymphatic system and peri- 
lymphatic interstitial tissue, interstitial thickening is seen on CT. The proliferation of these cells in combination with lymphatic dilatation contributes to this interstitial thickening (Fig. 8c) [21].

Sarcoidosis is a systemic entity characterised by the development of non-caseating granulomatous inflammation [22]. The most common parenchymal findings include irregular thickening of the bronchovascular bundles and small nodules in a perilymphatic distribution. Ground-glass attenuation and crazy-paving pattern are also described in sarcoidosis [1]. The linear pattern is caused by interstitial fibrosis.

Adult respiratory distress syndrome (ARDS) is a form of pulmonary oedema. Diagnosis is based on impaired diffusion capacity, reduced compliance of the lung and typical radiological findings. Chest $\mathrm{CT}$ features are bilateral consolidation and ground-glass attenuation [23]. Other findings such as reticular and linear opacities can also be seen. Histological features include oedema of the alveoli and perivascular spaces with filling of the alveoli by a protein-rich fluid $[22,24,25]$. The progress to architectural distortion and honeycombing with thickening of the inter- and intralobular septa is responsible for the linear accentuation.

The CT findings in patients with leukaemia consist mainly of ground-glass attenuation, centrilobular nodules and thickening of the bronchovascular bundles in the peripheral lung. The combination of ground-glass opacities and the thickening of the bronchovascular bundles can produce the crazy-paving pattern [26]. Accumulation of fluid in the alveolae causes the ground-glass opacification. Accumulation of fluid along the interlobular septa and along the walls of the alveolae can cause the periacinar pattern.

More than half of allogeneic haematopoietic stem cell transplant (HSCT) recipients develop graft-versus-host disease (GVHD), which remains a major cause of morbidity and mortality. HRCT findings in patients with GVHD are non-specific: diffuse interstitial and alveolar infiltrates are the most prominent features [27]. Depending the interstitial and alveolar component, a crazy-paving pattern can also be seen. On biopsy multiple hyaline membranes and fibroproliferative alterations can be seen, caused by the interstitial fibrosis and responsible for the linear pattern on CT. The multiple exudates into the alveolae are responsible for the ground-glass attenuation [28].

Organising pneumonia is a chronic inflammatory process characterised by plugs of granulation tissue in the lumen of distal small airways, often extending into the alveolar spaces, associated with an interstitial cellular response [29]. Typical CT features are scattered and asymmetric bilateral subpleural as well as peribronchovascular consolidation. A crazypaving pattern can be seen but is an uncommon finding [30].

Bronchioloalveolar carcinoma (BAC) has been classified into mucinous and non-mucinous subgroups and is characterised by a lepidic growth pattern through the airways and air spaces with preservation of the lung architecture. BAC may present with a variety of $\mathrm{CT}$ appearances. Features of $\mathrm{BAC}$ are the $\mathrm{CT}$ angiogram sign or air bronchograms in solitary nodules and in the periphery of larger consolidations, unifocal or multifocal ground-glass opacities, the crazy-paving pattern, and lobar or multilobar consolidation and cavitating nodules [31]. In patients with a crazy-paving pattern, the ground-glass attenuation reflects the lowdensity intra-alveolar material (glycoprotein), whereas the superimposed lines are due to infiltration of the interstitium by inflammatory or tumour cells [32].

\section{Conclusion}

The crazy-paving pattern on CT is a non-specific finding. It is characterised by scattered or diffuse areas of groundglass attenuation with superimposition of a linear pattern. This linear network can be caused by thickening of interlobular or intralobular septa or the presence of intralobular fibrosis, or it can be caused by a linear deposition of material within the airspaces. Most diseases can be diagnosed based on clinical and radiological findings. In a minority of cases a biopsy with histopathological examination is needed to establish the diagnosis.

\section{References}

1. Lee CH (2007) The crazy-paving sign. Radiology 243:905-906

2. Rossi SE, Erasmus JJ, Volpacchio M et al (2003) "Crazy-paving" pattern at thin-section $\mathrm{CT}$ of the lungs: radiologic-pathologic overview. Radiographics 23:1509-1519

3. Hansell DM, Bankier AA, MacMahon H et al (2008) Fleischner Society: glossary of terms for thoracic imaging. Radiology 246:697-722

4. Verschakelen JA, de Wever W (2007) Computed tomography of the lung. A pattern approach. Springer, Heidelberg

5. Akata S, Park J, Shindo H et al (2007) Barium aspiration showing crazy-paving appearance on high-resolution computed tomography. Australas Radiol 51(Suppl):B235-B237

6. da Silva Filho FP, Marchiori E, Valiante PM, Escuissato DL, Gasparetto TD (2008) AIDS-related Kaposi sarcoma of the lung presenting with a "crazy-paving" pattern on high-resolution CT: imaging and pathologic findings. J Thorac Imaging 23:135-137

7. Gasparetto EL, Tazoniero P, Escuissato DL et al (2004) Pulmonary alveolar microlithiasis presenting with crazy-paving pattern on high resolution CT. Br J Radiol 77:974-976

8. Johkoh $\mathrm{T}$, Itoh $\mathrm{H}$, Muller $\mathrm{NL}$ et al (1999) Crazy-paving appearance at thin-section CT: spectrum of disease and pathologic findings. Radiology 211:155-160

9. Choi HK, Park CM, Goo JM, Lee HJ (2010) Pulmonary alveolar proteinosis versus exogenous lipoid pneumonia showing crazypaving pattern: comparison of their clinical features and highresolution CT findings. Acta Radiol 51:407-412

10. Ishii H, Trapnell BC, Tazawa R et al (2009) Comparative study of high-resolution $\mathrm{CT}$ findings between autoimmune and secondary pulmonary alveolar proteinosis. Chest 136:1348-1355 
11. Zontsich T, Helbich TH, Wojnarovsky C, Eichler I, Herold CJ (1998) Pulmonary alveolar proteinosis in a child: HRCT findings before and after bronchoalveolar lavage. Eur Radiol 8:1680-1682

12. Laurent F, Philippe JC, Vergier B et al (1999) Exogenous lipoid pneumonia: HRCT, MR, and pathologic findings. Eur Radiol 9:1190-1196

13. Marchiori E, Zanetti G, Mano CM et al (2010) Lipoid pneumonia in 53 patients after aspiration of mineral oil: comparison of highresolution computed tomography findings in adults and children. J Comput Assist Tomogr 34:9-12

14. Bergin CJ, Wirth RL, Berry GJ, Castellino RA (1990) Pneumocystis carinii pneumonia: CT and HRCT observations. J Comput Assist Tomogr 14:756-759

15. Hartman TE (2003) The HRCT features of extrinsic allergic alveolitis. Semin Respir Crit Care Med 24:419-426

16. Lynch JPIII, Saggar R, Weigt SS, Zisman DA, White ES (2006) Usual interstitial pneumonia. Semin Respir Crit Care Med 27:634-651

17. Schmidt SL, Sundaram B, Flaherty KR (2009) Diagnosing fibrotic lung disease: when is high-resolution computed tomography sufficient to make a diagnosis of idiopathic pulmonary fibrosis? Respirology 14:934-939

18. Coche E, Weynand B, Noirhomme P, Pieters T (2001) Nonspecific interstitial pneumonia showing a "crazy paving" pattern on high resolution CT. Br J Radiol 74:189-191

19. Sumikawa H, Johkoh T, Ichikado K et al (2009) Nonspecific interstitial pneumonia: histologic correlation with high-resolution CT in 29 patients. Eur J Radiol 70:35-40

20. Murayama S, Murakami J, Yabuuchi H, Soeda H, Masuda K (1999) "Crazy paving appearance" on high resolution CT in various diseases. J Comput Assist Tomogr 23:749-752

21. Paslawski M, Krzyzanowski K, Zlomaniec J (2004) Lymphangitis carcinomatosa in thin section computed tomography. Ann Univ Mariae Curie Sklodowska Med 59:1-5
22. Collins J, Stern EJ (1997) Ground-glass opacity at CT: the ABCs. AJR Am J Roentgenol 169:355-367

23. Nobauer-Huhmann IM, Eibenberger K, Schaefer-Prokop C et al (2001) Changes in lung parenchyma after acute respiratory distress syndrome (ARDS): assessment with high-resolution computed tomography. Eur Radiol 11:2436-2443

24. Chan MS, Chan IY, Fung KH et al (2004) High-resolution CT findings in patients with severe acute respiratory syndrome: a pattern-based approach. AJR Am J Roentgenol 182:49-56

25. Maimon N, Paul N, Downey GP (2006) Progressive dyspnea associated with a crazy-paving appearance on a chest computed tomography scan. Can Respir J 13:269-271

26. Okada F, Ando Y, Kondo Y et al (2004) Thoracic CT findings of adult T-cell leukemia or lymphoma. AJR Am J Roentgenol 182:761-767

27. Liu QF, Luo XD, Ning J et al (2009) Association between acute graft versus host disease and lung injury after allogeneic haematopoietic stem cell transplantation. Hematology 14:63-72

28. Marchiori E, Escuissato DL, Gasparetto TD, Considera DP, Franquet T (2009) "Crazy-paving" patterns on high-resolution CT scans in patients with pulmonary complications after hematopoietic stem cell transplantation. Korean J Radiol 10:21-24

29. Epler GR, Colby TV, McLoud TC, Carrington CB, Gaensler EA (1985) Bronchiolitis obliterans organizing pneumonia. N Engl J Med 312:152-158

30. Muller NL, Staples CA, Miller RR (1990) Bronchiolitis obliterans organizing pneumonia: CT features in 14 patients. AJR Am J Roentgenol 154:983-987

31. Patsios D, Roberts HC, Paul NS et al (2007) Pictorial review of the many faces of bronchioloalveolar cell carcinoma. Br J Radiol 80:1015-1023

32. Akira M, Atagi S, Kawahara M, Iuchi K, Johkoh T (1999) Highresolution $\mathrm{CT}$ findings of diffuse bronchioloalveolar carcinoma in 38 patients. AJR Am J Roentgenol 173:1623-1629 